\title{
Local anaesthetic ropivacaine protects rats from myocardial ischaemia/reperfusion injury by inhibition of COX-2
}

\author{
Zhou $\mathrm{Yu}^{1}$, Sufang Sun ${ }^{2}$, Fang $\mathrm{Hu}^{1}$
}

${ }^{1}$ Department of Anesthesiology, The First People's Hospital, Fuyang, China 2Department of Obstetrics, The First People's Hospital, Fuyang Hangzhou, China

Submitted: 11 October 2020

Accepted: 16 January 2021

Arch Med Sci

DOI: https://doi.org/10.5114/aoms/132472

Copyright $\odot 2021$ Termedia \& Banach

\begin{abstract}
Introduction: Myocardial ischaemia/reperfusion (I/R) injury is the leading cause of morbidity and mortality worldwide. Despite novel advances in therapeutics, the management of myocardial I/R is still an unmet medical need. Therefore, in the present study, we have demonstrated the protective effect of ropivacaine (RPC) on the myocardial infarction in rats and its underlying mechanism.

Material and methods: Initially, the effect of RPC was determined on the infarct size and histopathology of cardiac tissues. The effect of RPC was also determined on the levels of various cardiac biomarkers such as creatine kinase $(C K)$, creatine kinase $M B(C K-M B)$, alanine aminotransferase (ALT), asparganine aminotransferase (AST), and lactate dehydrogenase (LDH), and biomarkers of oxidative stress (MDA, SOD, and GSH) and inflammation (tu-

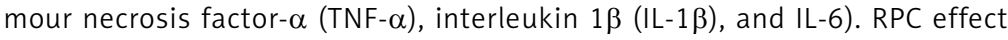
was also quantified on cellular apoptosis and COX-2 and iNOS expression via western blot analysis. The RPC was further docked into the active site of COX-2.

Results: It has been found that RPC reduces the improves haemodynamics of (LVSP and $\pm \mathrm{dp} / \mathrm{d} t_{\max }$, and LVEDP), infarct percentage and architecture of cardiac tissues of rats. It also reduces the level of studies cardiac injury biomarkers together with a reduction of oxidative stress (MDA, SOD, and GSH) and inflammation (TNF- $\alpha$, IL-1 $\beta$, and IL-6). Upon administration of RPC, the rate of cellular apoptosis was found to be greatly reduced, with a reduction in COX-2 and iNOS expression. In docking analysis, RPC creates van der Waals forces and pi-interactions with Tyr381, Arg106, Val102, Leu345, Val509, Ser339, Leu338, Val335, Ala513, His75, and Leu517 at the catalytic site of COX-2.

Conclusions: Collectively, our results demonstrated that ropivacaine showed significant benefit against myocardial ischaemic injury.
\end{abstract}

Key words: ropivacaine, oxidative stress, inflammation, apoptosis, docking, COX-2.

\section{Introduction}

Despite various advances in diagnostics and therapeutics, myocardial infarction is still posing a significant threat to mankind. It remains a leading cause of morbidity and mortality throughout the world [1]. Dramatic

\author{
Corresponding author: \\ Zhou Yu PhD \\ Department of \\ Anesthesiology \\ The First People's Hospital \\ of Fuyang, China \\ E-mail: 254897547@qq.com
}


changes in lifestyle and eating habits predispose a significant number of individuals to myocardial infarction across the globe in the comingyears [2,3]. Particularly in China, as per the Chinese disease report published in 2020, cardiovascular diseases account for approximately $45 \%$ of deaths in China $[4,5]$. The loss of blood supply to the heart is the main factor for myocardial infarction, which leads to ischaemia of the cardiomyocytes and subsequent necrosis of cardiac tissue [6]. Thus, the majority of drugs used against myocardial ischaemia have been aimed to restore the lost blood supply to prevent infarction, by a combination of agents, which include vasopressor agents, calcium channel antagonists, antiarrhythmic agents, antihypertensive agents, angiotensin convertase enzyme (ACE) inhibitors, and vasodilator. This signifies that none of the single agents can be used to control myocardial infarction and associated ischaemia. Moreover, the restoration of blood supply is not the only factor that can improve the prognosis of patients, but subsequent reperfusion also worsens the situation $[7,8]$. This causes the injury to the myocardial tissues known as myocardial ischaemia/reperfusion injury. The generation of reactive oxygen species (ROS) in the reperfusion stage promotes oxidative stress due to the disparity between antioxidant defence mechanisms. It also promotes lipid peroxidation, tissue infiltration, alteration in vascular permeability, and production of various inflammatory cytokines that lead to inflammation [9-11]. Therefore, studies have proven the effectiveness of antioxidants and anti-inflammatory agents in MI/R injury [12-14].

Studies have shown the importance of local anaesthetics (LA) as antiarrhythmic agents. Some clinically relevant LAs are established antiarrhythmic agents, such as lignocaine, which provides an antiarrhythmic effect via inhibition of cardiac sodium channel [15], procainamide impairs the myocardial contractile force and lowers cardiac output and systemic arterial pressure to provide antiarrhythmic effects [16], and prilocaine prevents aconitine-induced arrhythmias [17]. Another LA, bupivacaine, in isolated cardiac tissues, decreases intra-cardiac conduction velocity and contractile force and depresses spontaneous sinoatrial activity. It also decreases cardiac output, myocardial contractility, and intra-cardiac conduction velocity, as shown by increased PR and QRS durations in anaesthetized animals [18]. However, it was later found to possess significant cardiotoxicity, which limits its use against myocardial diseases [19, 20]. Thus, it could be suggested that the antiarrhythmic action of LA is similar to local anaesthesia, in that they prevent the generation of action potential mainly by blocking voltage-gated $\mathrm{Na}^{+}$channels, $\mathrm{Ca}^{2+}$ channels, and $\mathrm{K}^{+}$channels, which results in membrane stability $[21,22]$. Ropivacaine (RPC) is an amide-based non-cardiotoxic local anaesthetic used during surgery, labour, and post-operative pain in adults and children [23, 24]. It blocks a signalling cascade related to tumour necrosis factor $\alpha$ (TNF- $\alpha)$, which results in the protection of endothelium [25]. It also protects experimental acute lung injury induced by bacterial lipopolysaccharide (LPS) via reduction of inflammation [26, 27]. The excellent anticancer activity of RPC was identified against cervical, gastric, and hepatic cancer cells [28-31]. Prompted by the above, the present study intended to investigate the effect of RPC on myocardial infarction in rats and its underlying mechanism.

\section{Material and methods}

\section{Animals}

Adult male Sprague-Dawley (8-10 weeks, 240-270 g) rats were obtained from the institutional animal house and kept in a strict hygienic and controlled laboratory environment with an ad libitum supply of food and water. The experiment was duly approved by the institutional ethical committee of The First People's Hospital of Fuyang Hangzhou and was performed according to the national guidelines of animal care and use of China.

\section{Initiation of experimental myocardial ischaemia/reperfusion (I/R) injury}

To establish myocardial I/R injury, the rats were fasted for $12 \mathrm{~h}$ before and had free access to water. The rats showing abnormal ECG were excluded from the experiment, and only normal rats were selected for further experiments. The selected rats were anaesthetized with $1.5 \mathrm{ml} / \mathrm{kg}$ sodium pentobarbital $(30 \mathrm{~g} / \mathrm{l})$ by i.p. subjected to the tracheal cannula. The breathing frequency of rats was maintained at 50-60 beats per minute using an ALC-V model animal ventilator with continuous monitoring by ECG. A $2-\mathrm{cm}$ longitudinal incision was made on the chest of rats, where the sternum muscle was fixed with a haemostatic clamp. A small orifice was created at the intercostal region of the $2^{\text {nd }}-3^{\text {rd }}$ ribs in the left side near the sternum. The heart of the rat was exposed after removing the pericardium, and a needle and thread were inserted at the lower end of the left atrial appendage to ligate the left anterior descending artery (LAD). The increase in ST-segment showed the successful creation of the MI model. The successful ligation showed reduced blood pressure and prominent ECG changes in rats. After reperfusion, local red colour was shown, and depression of ST-segment was observed on ECG. The chest of the control group rats was opened 
and threaded without ligation. In the I/R group the rats were subjected to ligation of the left ventricle for $30 \mathrm{~min}$, and then reperfusion for $120 \mathrm{~min}$. In the treatment group, RPC was administered 30 min before the ischaemia followed by a 5 -min interval, and ligation was performed for $30 \mathrm{~min}$ with subsequent reperfusion for $120 \mathrm{~min}$.

\section{Experimental design}

Thirty SD rats were taken and randomly divided into 5 groups containing 6 animals in each group.

- group 1: normal control;

- group 2: IR rats;

- group 3: IR + RPC (10 mg/kg);

- group 4: IR + RPC (15 mg/kg);

- group 5: IR + RPC (20 mg/kg).

Different doses of RPC as indicated above were administered immediately through intraperitoneal injection after being dissolved in $0.9 \% \mathrm{NaCl}$ including 1\% DMSO 30 min before the myocardial ischaemia models were completed. All rats then received their respective treatments once daily until the end of the experiment. Thereafter, the rats were killed under anaesthetized conditions using urethane (1 $\mathrm{g} / \mathrm{kg}$, intra-peritoneally injected) to isolate blood. The heart tissues were washed in ice-cold normal saline after harvesting.

\section{Assessment of haemodynamic and cardiac function}

The left ventricular systolic pressure (LVSP) and left ventricular end-diastolic pressure (LVEDP) were recorded at the start of the experiment, before ischaemia, after 30-min ischaemia, and after 120-min reperfusion. Values of $+\mathrm{dp}_{\mathrm{ddt}}$ and $-\mathrm{dp} /$ $\mathrm{d} t_{\max }$ were calculated using the analysing system of a RM6240 multichannel physiological signal detector.

\section{Determination of infarct size}

To determine myocardial infarct area the Even's blue-2,3,5-triphenyl tetrazolium chloride (TTC) method was used. Briefly, the Evans blue was administered into the tail vein, and non-ischaemic myocardial tissues was coloured dark blue. At this stage, the hearts were excised from the rats and weighed after drying on filter paper. The LV was excised from the heart, weighed, frozen in for $1 \mathrm{~h}$ at $-20^{\circ} \mathrm{C}$, and then sliced into 6 slices along the axis. These slices were then placed into $1 \%$ TTC at pH 7.4 and incubated at $37^{\circ} \mathrm{C}$ followed by soaking in $10 \%$ formaldehyde for $15 \mathrm{~min}$. The slices were then photographed, and their weight was recorded.

The infarct size was observed as a grey-white colour due to the absence of dehydrogenase in dead cells, which is unable to stain because dead cells cannot reduce TTC to a deep red colour. The myocardial ischaemic area was measured as per the earlier reported procedure [32].

\section{Determination of serum LDH and CK levels}

The serum concentration of myocardial enzymes, lactate dehydrogenase (LDH), and creatine kinase myocardial band (CK) was recorded using marketable assay kits as per the instructions provided, using a microplate reader at $340 \mathrm{~nm}$. (Nanjing Jiancheng Bioengineering Institute, China).

\section{Evaluation of lipid peroxidation and antioxidant enzyme levels}

The MDA level, SOD, and GSH activities in the heart homogenate supernatant was determined as per the supplier's instructions, using a microplate reader at 560 and $532 \mathrm{~nm}$ (Nanjing Jiancheng Bioengineering Institute, China).

\section{Determination of TNF- $\alpha$, IL-1 $\beta$, and IL- 6 levels in cardiac tissues}

The serum level of tumour necrosis factor- $\alpha$ (TNF- $\alpha$ ) and interleukin (IL) 6 was determined by commercial ELISA kits as per the supplier's instructions, using a microplate reader at $450 \mathrm{~nm}$ (Nanjing Jiancheng Bioengineering Institute, China).

\section{Myocardial cell apoptosis}

Briefly, the cells were lysed with trypsin and washed twice with PBS. $20 \mu \mathrm{l}$ Annexin-V-FITC labelling solution was added to $1 \mathrm{ml}$ buffer solution, and then $20 \mu \mathrm{l} \mathrm{PI}$ reagent was added to the resulting cell after lysing. The cells were then kept at room temperature without light for $5 \mathrm{~min}$. The apoptosis was measured by flow cytometer FACScan flow cytometer (Becton Dickinson Company, USA) using CellQuest software (ver 4.0; BD Biosciences).

\section{Western blot analysis}

The isolated protein was loaded on SDS-PAGE (sodium dodecyl sulphate-polyacrylamide gel electrophoresis) and shifted onto the (polyvinylidene difluoride) PVDF membrane and probed with primary antibody. Following incubation with HRP-conjugated goat anti-rabbit IgG for $1 \mathrm{~h}$ at room temperature, the protein bands were analysed using an enhanced chemiluminescence reagent by using ImageJ v1.42q software (National Institutes of Health, Bethesda, MA, USA).

\section{Docking study}

The docking study was conducted using ropivacaine as the ligand into the 3D crystal structure of the COX-2 protein model. The CDOCKER 
protocol of Discovery Studio (3.0) was selected to perform this study. The protein preparation, ligand preparation, active site identification, and docking were performed using the default setting of the CDOCKER protocol as per the manufacturer's instruction. The receptor-ligand interaction of topranked posed of ropivacaine was visualized using suitable scripts of the software. Moreover, the 2D interaction diagram was constructed using 2D interaction viewer commands.

\section{Ethical approval}

The study was approved by the First People's Hospital of Fuyang Hangzhou. All experiments were conducted under internationally accepted principles for laboratory animal use and care, as found in the United States guidelines $(\mathrm{NIH}$ publication no. 85-23, revised in 328 1985).

\section{Statistical analysis}

Data were analysed by SPSS 17.0 software and expressed as the means \pm SD. Differences were analysed by one-way analysis of variance (ANOVA) followed by Dunnett's test for individual comparisons between each group mean. A $p$-value of 0.05 was considered statistically significant.
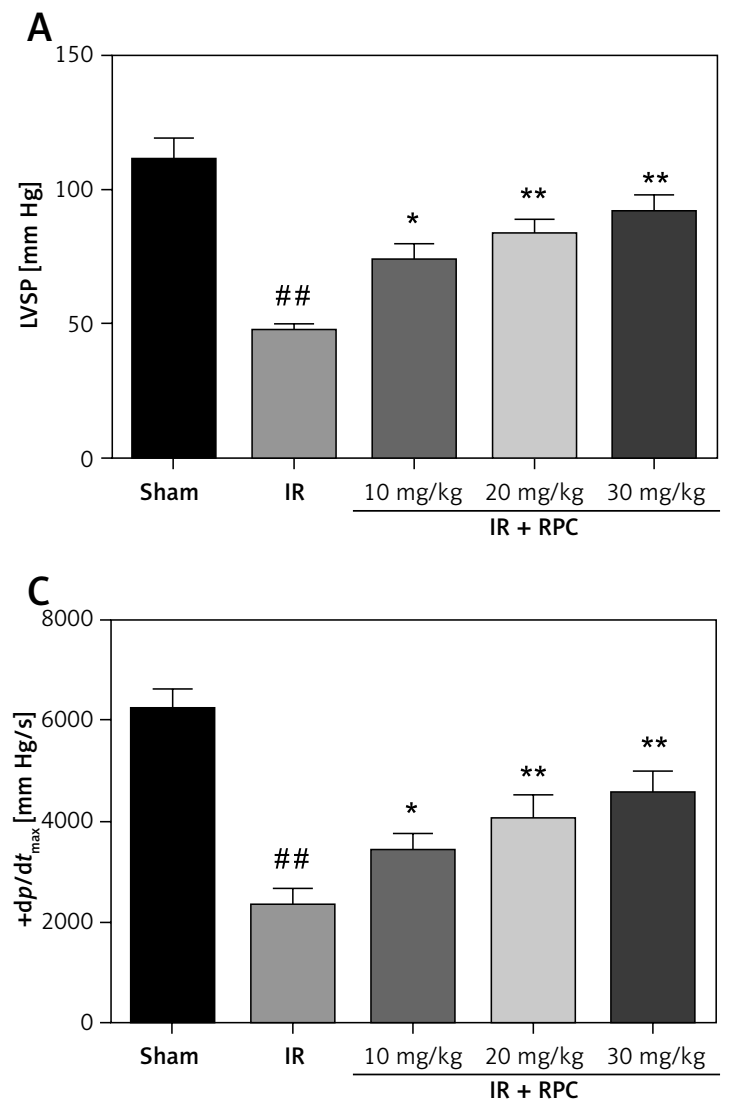

Figure 1. Effect of RPC on the haemodynamic parameter after MI/R in rats. A - LVSP, B - LVEDP, C $-+d p / d t$ D $--d p / d t$. Data expressed as means \pm SDs. ${ }^{\#} p<0.05$ compared with sham group; ${ }^{*} p<0.05,{ }^{* *} p<0.01 \mathrm{com}-$ pared with I/R group

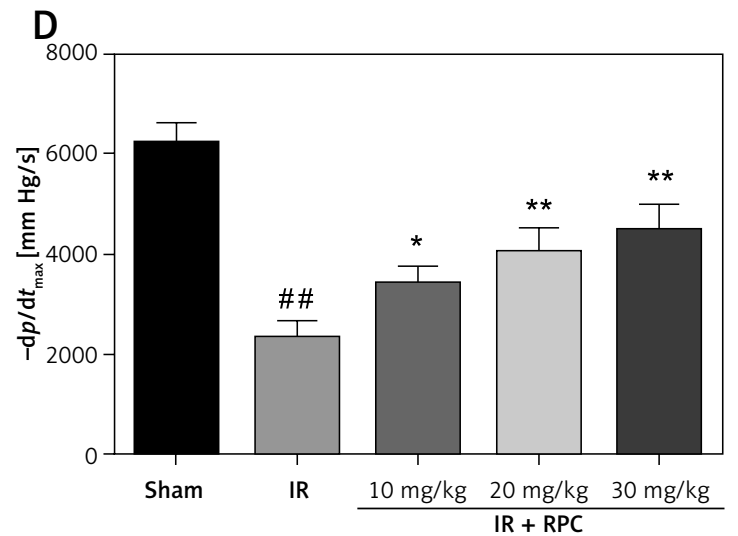

\section{The effect of RPC on the haemodynamic parameters}

The effect of RPC was first investigated on the haemodynamic parameters in anaesthetized rats. The results are shown in Figure 1. After myocardial I/R injury, the disease control rats showed a decreased level of LVSP and $\pm d p / d t_{\text {max }}$, and LVEDP was found to be significantly increased as compared to the sham. These effects were partly restored to near normal after treatment with RPC in a concentration-dependent manner. These results were further substantiated with ECG analysis of rats following myocardial ischaemia-reperfusion injury (supplementary Figure S1). It was found that pre-treatment of RPC caused a significant reduction in the ST-segment of rats as compared to IR rats.

\section{The effect of RPC on the myocardial infarct area}

As shown in Figure $2 \mathrm{~A}$, the infarct size was found to greatly increased in the IR group with no treatment as compared to the sham. The RPC-treated group showed a significant reduction in the infarct size of the animals in a dose-de-

B

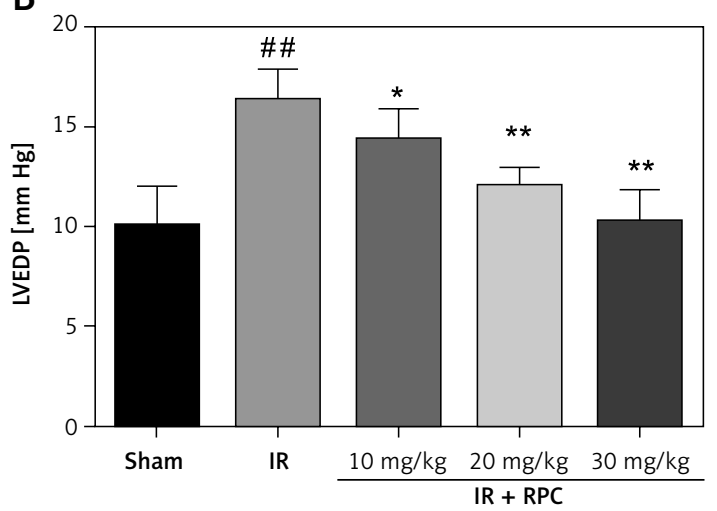




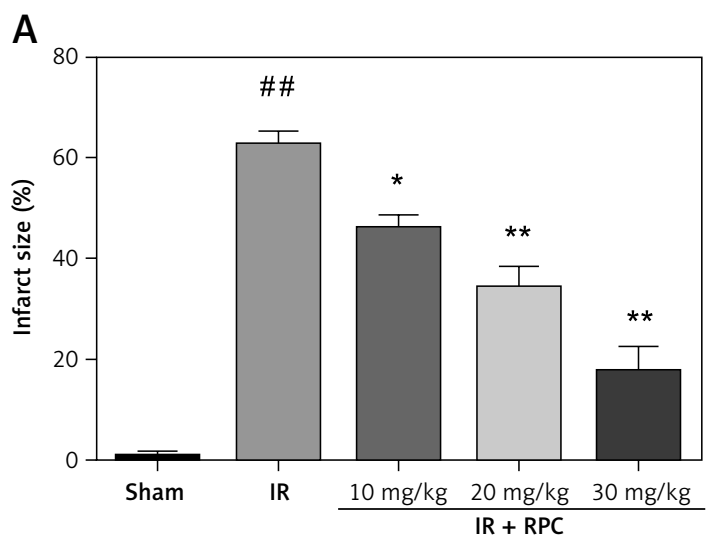

B

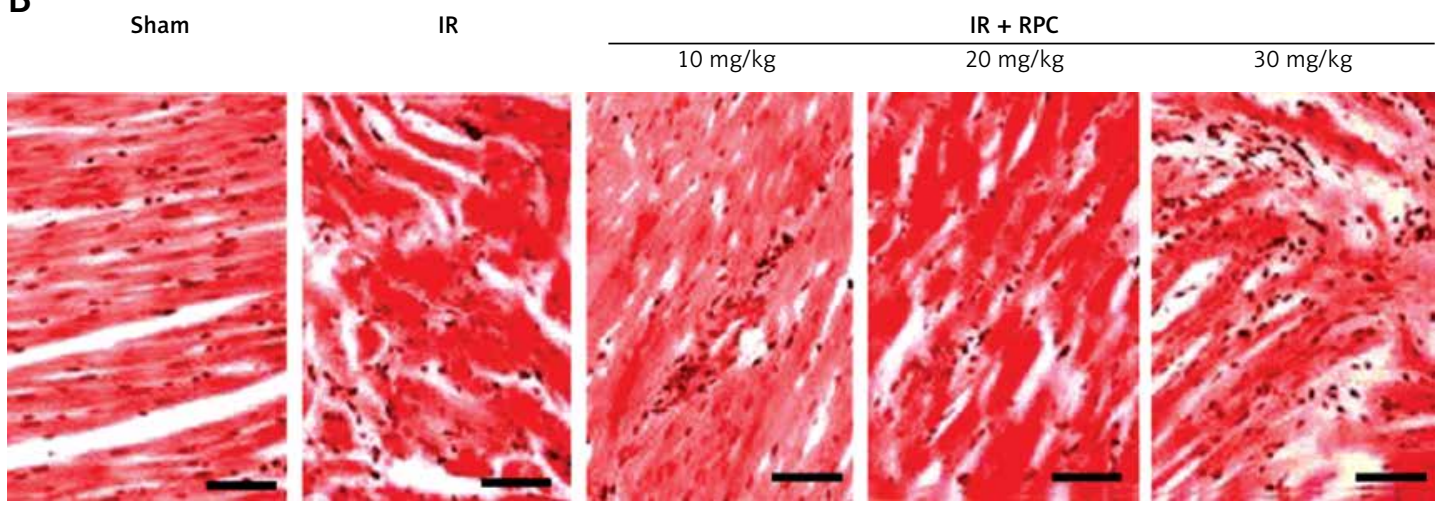

Figure 2. Effect of RPC on the (A) infarct volume and (B) histopathology of myocardial tissue by $\mathrm{H}$ and $\mathrm{E}$ analysis. Data were expressed as means \pm SD. ${ }^{\# \#} p<0.05$ compared with sham group; ${ }^{*} p<0.05$, ${ }^{* *} p<0.01$ compared with I/R group

pendent manner in comparison to the IR control group.

\section{The effect of RPC on the histopathology of cardiac tissues}

As shown in Figure $2 \mathrm{~B}$, the IR group showed marked necrosis and inflammation of myocardial tissues, which was absent in the sham-treated group. However, upon administration of RPC, these histopathological changes were returned near to normal, as confirmed by reduced oedema, necrosis, and inflammation in a dose-dependent manner. This observation confirmed that RPC would be able to mitigate the after-effects of MRI in rats.

\section{The effect of RPC on the cardiac injury biomarkers}

The effect of RPC was further evaluated on various biomarkers of cardiac injury, and the results are presented in Figure 3. It was found that the concentrations of various tested biochemical mediators (e.g. CK, CK-MB, ALT, AST, and LDH) were highly elevated in the IR group, which directly correlates with the myocardial injury as compared to the sham. The RPC-treated group showed dose-dependent reduction of the serum level of the tested biomarkers as compared to the IR group. This signifies the protective behaviour of RPC against IR injury.

\section{The effect of RPC on oxidative stress biomarkers}

The effect of RPC was assessed on numerous biomarkers of oxidative stress, as presented in Figure 4. The level of MDA was found to be elevated, together with a reduction in SOD and GSH in the IR group, as compared with the sham group. Moreover, upon administration of RPC, the level of these biomarkers depicting oxidative stress was significantly restored approximately near to normal in a dose-dependent manner.

\section{The effect of RPC on the pro-inflammatory cytokines}

As shown in Figure 5, the level of cytokines such as TNF- $\alpha$, IL-1 $\beta$, and IL- 6 was found to be markedly increased in the IR group as compared to the controls. However, the levels of these tested cytokines were found to be significantly reduced in the RPC-treated group in a dose-dependent manner. Thus, it could be suggested that RPC has 

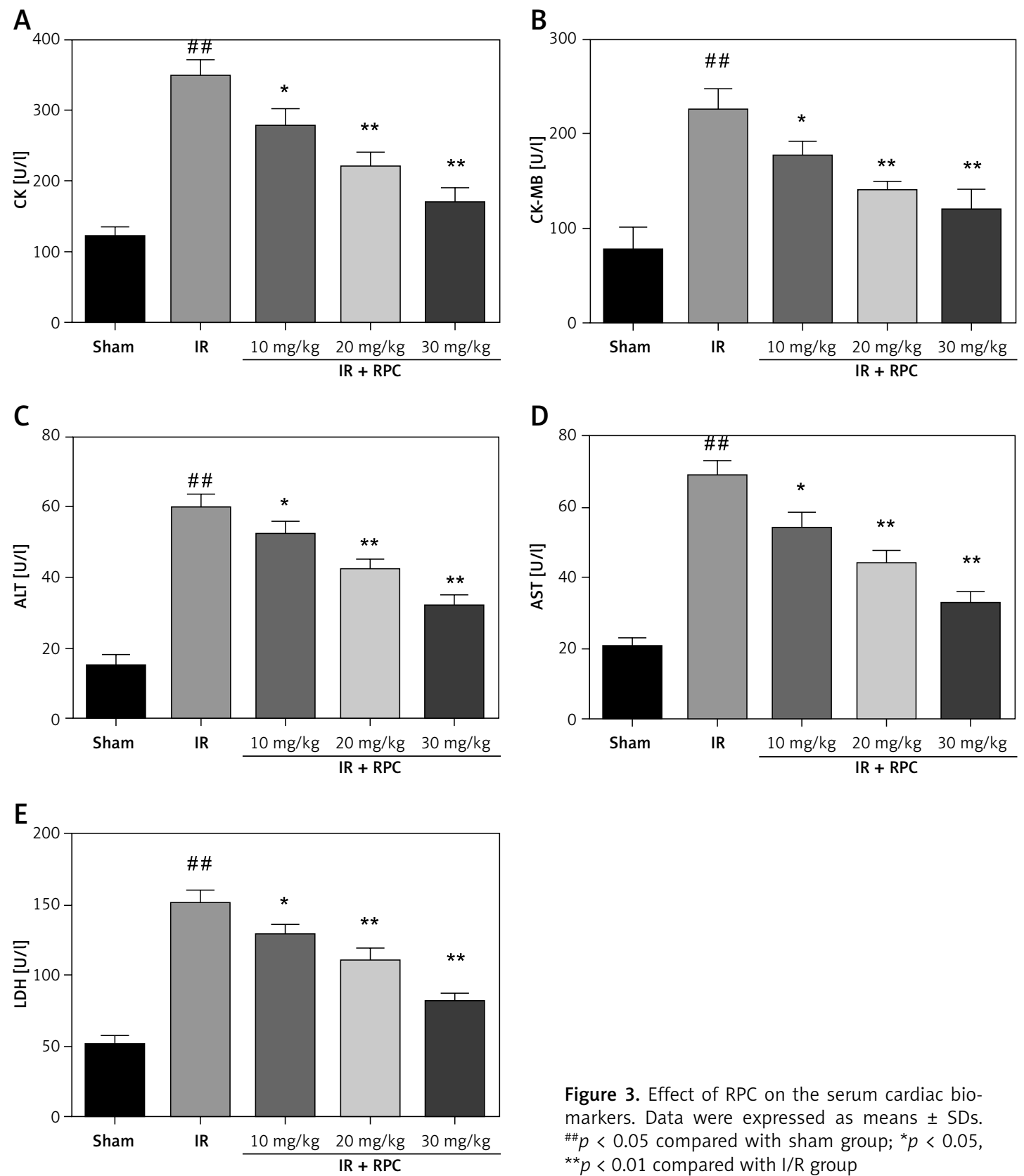

an inhibitory effect on the hyperactivated pro-inflammatory cytokines.

\section{The effect of RPC on cellular apoptosis}

As shown in Figure 6, results suggested that, as compared to sham, the rate of cell apoptosis was found to be highly increased in the IR disease group, whereas in the RPC-treated group, the rate of cellular apoptosis was found to be greatly reduced.

The effect of ropivacaine on COX-2 and iNOS by western blot analysis

As expected, the IR group with no treatment showed an elevated level of these 2 proteins in

Figure 3. Effect of RPC on the serum cardiac biomarkers. Data were expressed as means \pm SDs. ${ }^{\# \#} p<0.05$ compared with sham group; ${ }^{*} p<0.05$, ${ }^{* *} p<0.01$ compared with I/R group

comparison to the sham. The RPC treated group, in a dose-dependent manner, showed a drastic drop in the level of COX-2 and iNOS (Figure 7). These results suggest that RPC might exert a cardioprotective effect via strong anti-inflammatory activity.

\section{Docking analysis of ropivacaine with COX-2}

In docking analysis, RPC was found to be deeply buried into the active site of the COX-2 protein structure by interacting with key catalytic residues (Figure 8). To further elaborate these interactions, a $2 \mathrm{D}$ interaction diagram of RPC with COX-2 was generated and displayed in Figure 9. It was found that RPC creates numerous interactions with 
A
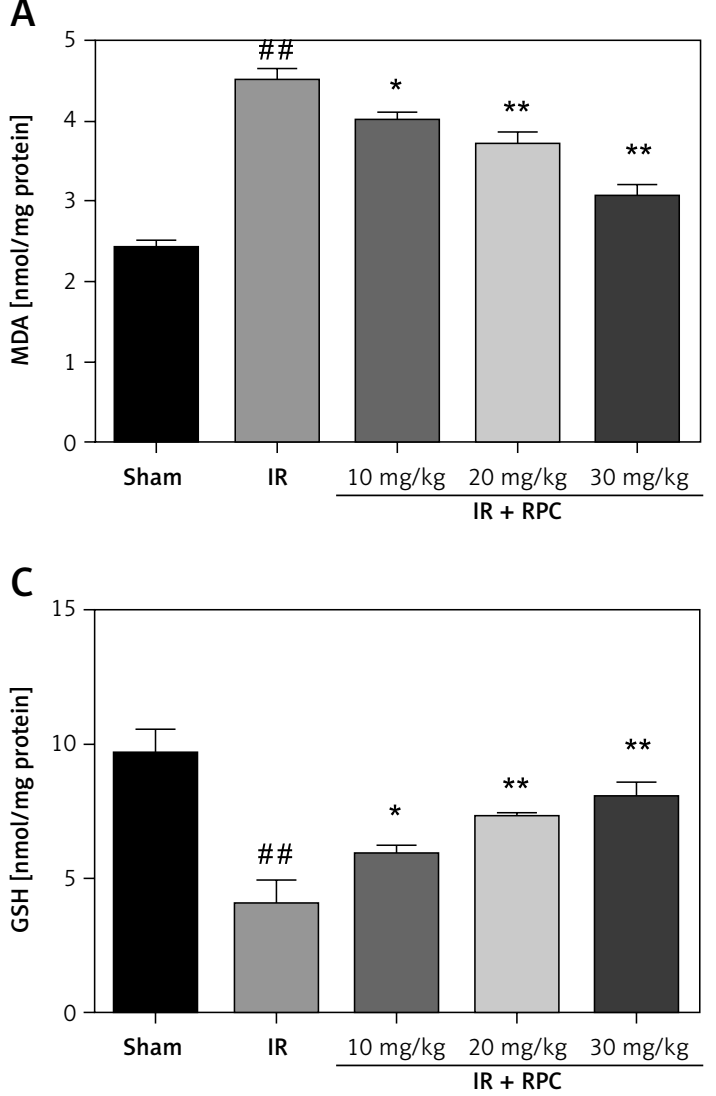

A

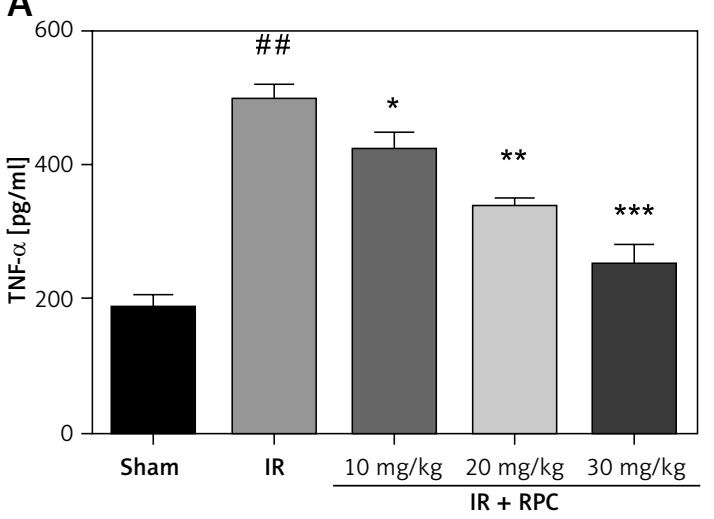

C

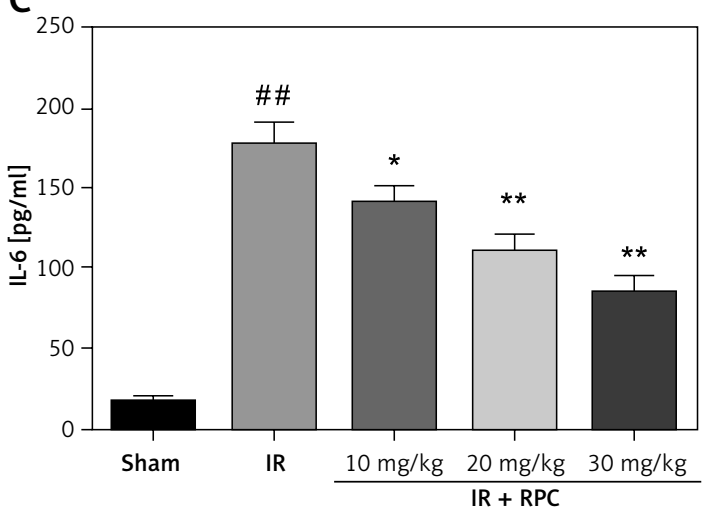

B

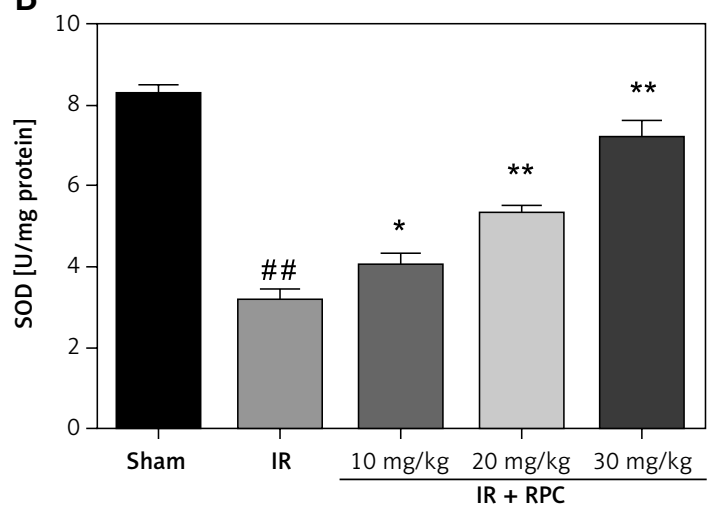

Figure 4. Effect of RPC on the various indices of oxidative stress. Data were expressed as means \pm SDs. ${ }^{\# \#} p<0.05$ compared with sham group; ${ }^{*} p<$ $0.05,{ }^{* *} p<0.01$ compared with I/R group

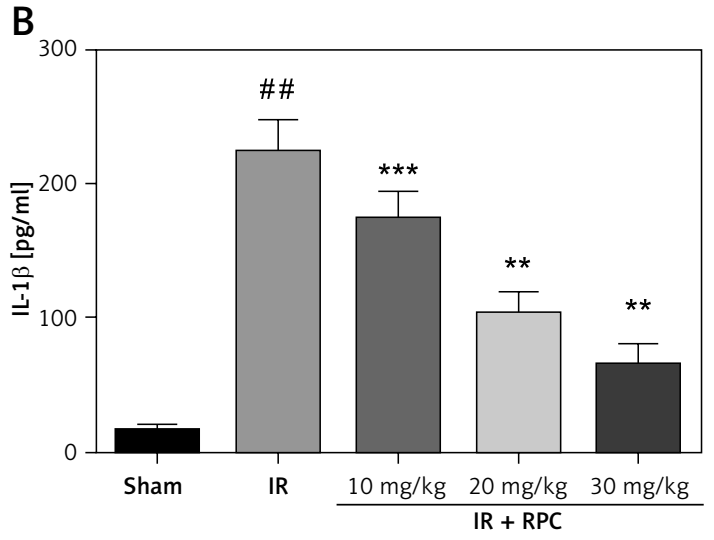

Figure 5. Effect of RPC on the serum level of pro-inflammatory cytokines. Data were expressed as means \pm SDs. ${ }^{\# \#} p<0.05$ compared with sham group; ${ }^{\star} p<0.05,{ }^{\star *} p<0.01$ compared with I/R group 
A
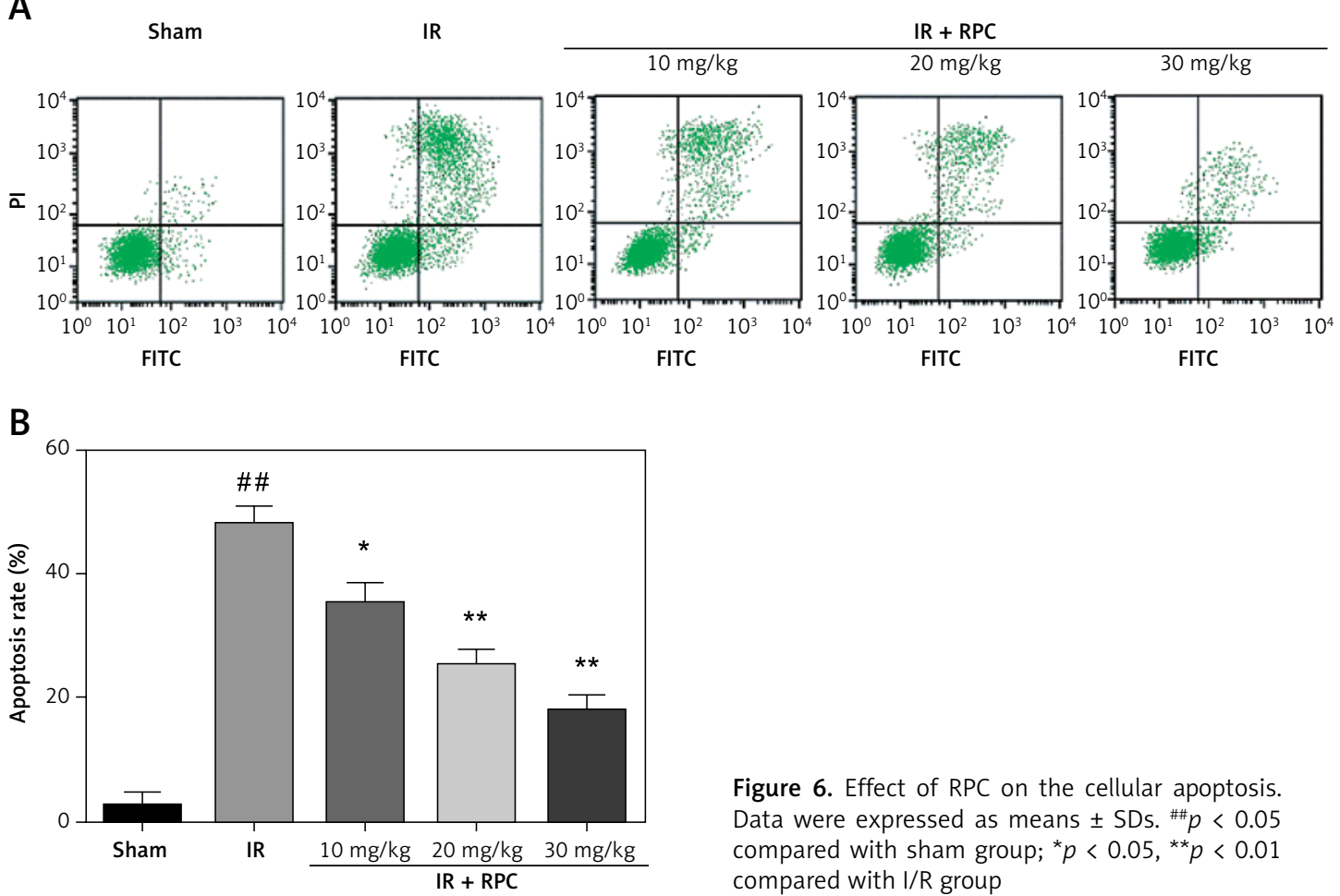

Figure 6. Effect of RPC on the cellular apoptosis. Data were expressed as means \pm SDs. \# $p<0.05$ compared with sham group; ${ }^{*} p<0.05,{ }^{* *} p<0.01$ compared with I/R group

A
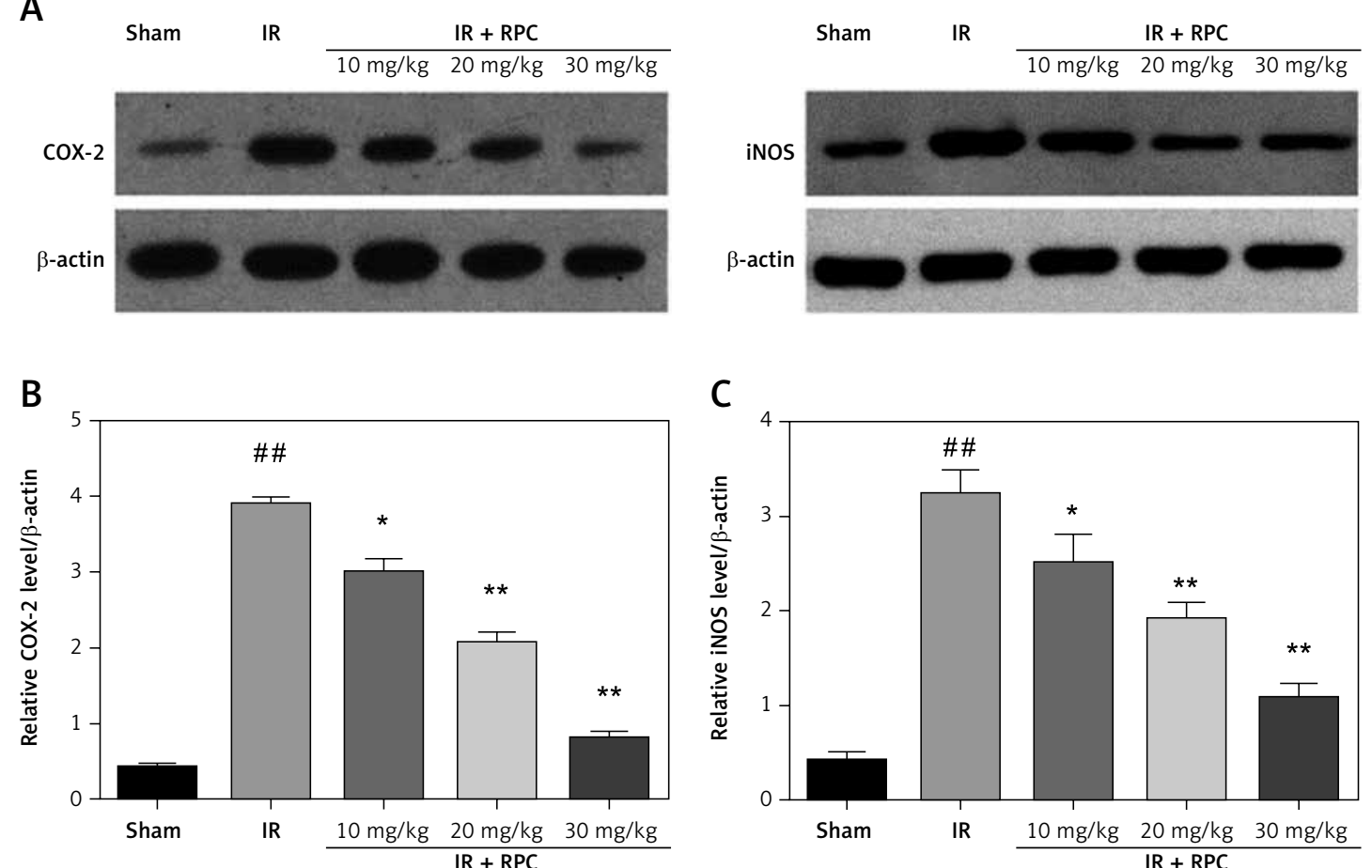

Figure 7. Effect of RPC on the expression of COX-2 and iNOS by western blot analysis. Data were expressed as means \pm SDs. ${ }^{\# \#} p<0.05$ compared with sham group; ${ }^{*} p<0.05,{ }^{* *} p<0.01$ compared with I/R group 


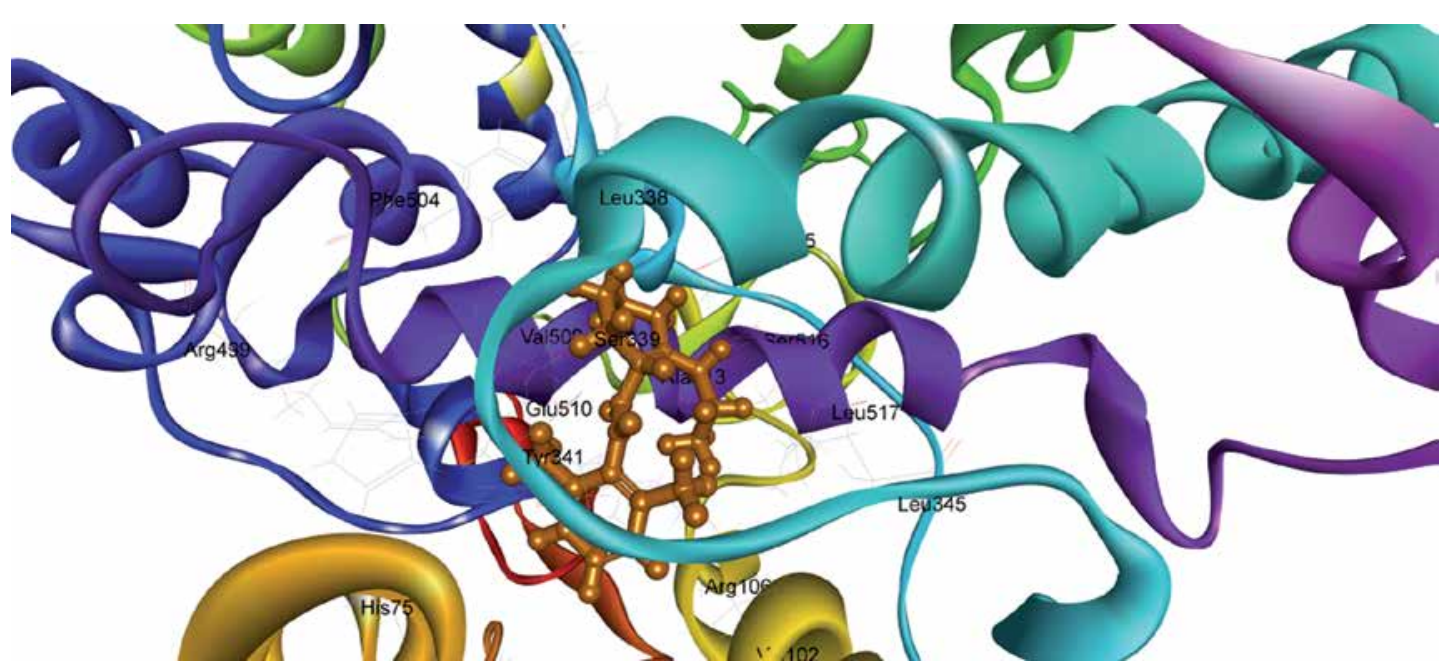

Figure 8. 3D orientation of RPC (shown in brown ball and stick) into the catalytic site of COX-2 (shown in ribbon)

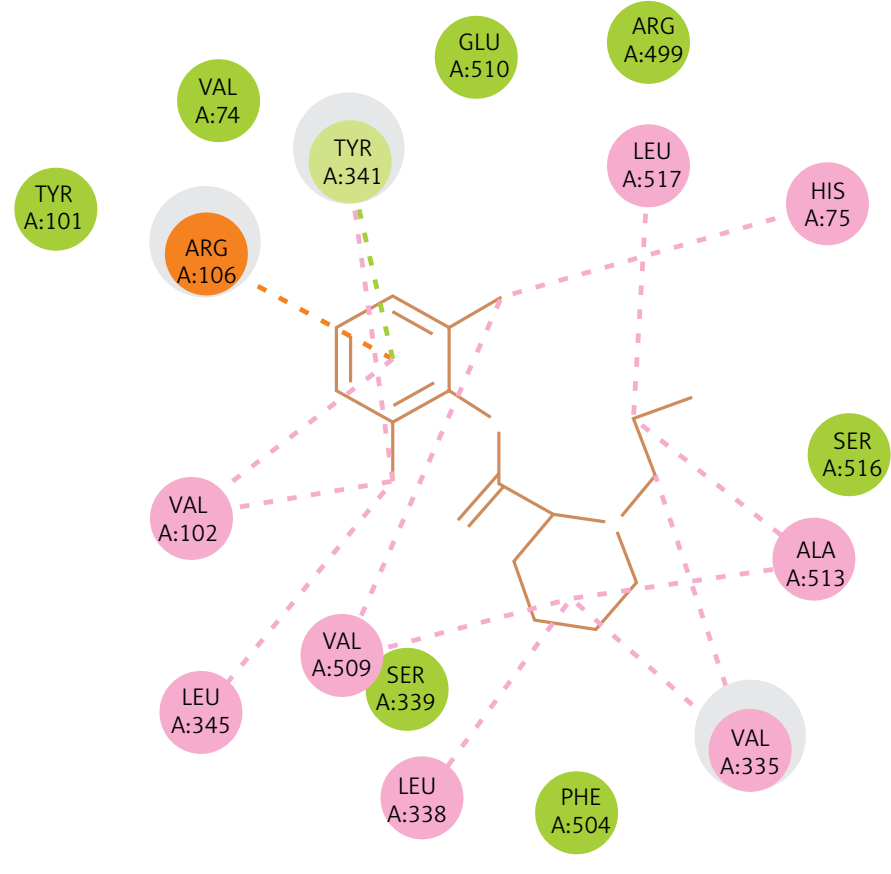

Interactions

$\square$ vander Waals $\quad \square$ Pi-Cation $\quad \square$ Pi-Lone Pair $\quad \square$ Pi-Pi T-shaped $\quad \square$ Alkyl $\quad \square$ Pi-Alkyl

Figure 9. 2D orientation of RPC into the catalytic site of COX-2

neighbouring residues via the formation of many van Der Waals and pi-interactions with Tyr381, Arg106, Val102, Leu345, Val509, Ser339, Leu338, Val335, Ala513, His75, and Leu517. The interaction shown by RPC was found to be similar to previous studies [33]. These strong interactions of RPC with the catalytic site of COX-2 provide the basis for its inhibitory activity against COX-2.

\section{Discussion}

Myocardial ischaemia/reperfusion injury is a devastating illness that impacts millions of in- dividuals, either directly as a patient or indirectly as a caregiver or family member of an affected individual. It creates a huge economic burden on the patients and their families due to long hospital stays. Thus, new agents are continuously developed towards finding a cheap and economic way to deal with it. In our present study, we have successfully demonstrated the protective effect of ropivacaine against experimentally induced myocardial ischaemia/reperfusion injury in SD rats. We have selected male SD rats because extra oestrogen treatment may result in reduced 
infarct size [34]. Secondly, accumulating evidence suggests that the infarct area in females is smaller than in males relative to body mass [35]. It has also been found that extra preconditioning and other cardio-protective approaches do not have any potential beneficial effect in females [36]. The interactions between cardioprotective signalling pathways in female animals and pre-and post-conditioning signalling pathways might be the reason for this effect. Infarction is the main prognostic factor in I/R injury. Studies showed that many novel agents provide a beneficial effect against IR injury via reduction of the infarct size. It has been shown that the infarct reducing the ability of any agent is directly correlated with its protective effect against myocardial IR injury [37-39]. Initially, the effect of RPC was investigated on the haemodynamic parameter of MI/R injury in rats. It was found that RPC restored the level of LVSP and $\pm \mathrm{dp} / \mathrm{dt}$ max, and LVEDP near to normal in a concentration-dependent manner. The ST-segment elevation is a characteristic hallmark of ischaemia/reperfusion injury, thus agents reducing ST-segment provide a beneficial effect against MI/R injury [40, 41]. In ECG analysis, RPC reduces ST-segment in rats. The effect of RPC was investigated on the infarct size of IR animals. It has been found that RPC significantly reduces infarct size. Accumulating evidence suggests that the utility of histopathological examination of myocardial tissue is a significant parameter for the assessment of the protection of chemicals against IR injury. It is a widely accepted methodology that allows the investigator to examine the cardiac tissue to assess the rate of improvement after the treatment [42-46]. Thus, we aimed to analyse the histopathology of cardiac tissues by $\mathrm{H}$ and $\mathrm{E}$ staining after administration of RPC to macroscopically visualize its cardioprotective effect. It was found that RPC causes improvement in the cardiac histopathology of animals. Cardiac injury biomarkers (LDH and MB) play a significant role in diagnostics, which allows the planning of a therapeutic regime during the time of injury or after the injury. It provides a suitable and efficient way to evaluate heart function. Compelling studies have suggested that after I/R injury the levels of these biomarkers were highly deregulated in direct proportion to the extent of I/R injury [47-49]. In the present study, RPC caused a significant reduction in cardiac injury biomarkers. Various evidence suggests that oxidative stress has a highly deleterious effect on the recovery from myocardial ischaemia/reperfusion injury. It induces the production of an excessive amount of reactive oxygen species to the impaired antioxidant system. These radicals are supposed to cause the annihilation of proteins, DNA, and lipids, simultaneously damaging the membrane to induce cell death [50-52]. Thus, agents improving the antioxidant system showed benefit against myocardial ischaemia/reperfusion. The RPC causes significant improvement of the antioxidant system after I/R injury, which was found following earlier studies. Inflammation in the hallmark of myocardial I/R injury. It is fuelled by the production of reactive oxygen species, which in turn promote oxidative stress and initiate a cascade of inflammatory response via recruitment of various pro-inflammatory cytokines [53, 54]. In the present study, RPC caused a reduction of the serum level of various pro-inflammatory cytokines. Myocardial apoptosis is greatly increased after IR injury and leads to the necrosis of myocardial tissue. Therefore, it is important to assess the effect of RPC on myocardial tissue [55, 56]. Towards this end, annexin V-FITC/PI double staining and flow cytometry was undertaken to analyse the effect of RPC on the apoptosis of myocytes, and it was found that RPC reduced apoptosis in a dose-dependent manner. To comprehend the underlying mechanism of the strong anti-inflammatory effect of RPC, which might be the reason for its protective effect against IR injury, we next aimed to investigate its effect on the expression of COX-2 and iNOS by western blot analysis. These were believed to play a vital role in the progression of inflammation after injury $[57,58]$. It was found that RPC causes a drastic decrease in the level of COX-2 and iNOS. Docking is a widely recognized and powerful tool in drug discovery to define the probable structural contacts of ligands with the protein of interest. It accelerates the process of drug discovery and helps in the discovery and optimization and preclinical tests [59-63]. Therefore, to provide the basis for strong COX-2 inhibitory activity of RPC, it was docked onto the catalytic site of the 3D-crystal structure of COX-2. These strong interactions of RPC with the catalytic site of COX-2 provide the rationale for its inhibitory activity against COX-2. Further research is required to investigate these in larger, longer-term studies.

In conclusion, our study has demonstrated the usefulness of ropivacaine against myocardial ischaemia/reperfusion injury. Ropivacaine showed a protective effect against myocardial injury possibly via amelioration of oxidative stress and apoptosis of myocardial tissue. Ropivacaine reduces inflammation in animals possibly via strong inhibition of COX-2 and iNOS. However, more studies are needed to provide the clinical basis of ropivacaine in myocardial injury.

\section{Conflict of interest}

The authors declare no conflict of interest. 


\section{References}

1. Mensah GA, Roth GA, Fuster V, The Global Burden of Cardiovascular Diseases and Risk Factors: 2020 and Beyond. J Am Coll Cardiol 2019; 74: 2529-32.

2. Reed GW, Rossi JE, Cannon CP. Acute myocardial infarction. Lancet 2017; 389: 197-210.

3. Voight BF, Peloso GM, Orho-Melander M, et al. Plasma HDL cholesterol and risk of myocardial infarction: a mendelian randomisation study. Lancet 2012; 380: 572-80.

4. Ma LY, Chen WW, Gao RL, et al. China cardiovascular diseases report 2018: an updated summary. J Geriatr Cardiol 2020; 17: 1-8.

5. Lu J, Zhang L, Lu Y, et al. Secondary prevention of cardiovascular disease in China. Heart 2020; 106: 1349-56.

6. Anderson IL, Morrow DA. Acute myocardial infarction. N Engl J Med 2017; 376: 2053-64.

7. Thiele H, Ohman EM, Desch S, et al. Management of cardiogenic shock. Eur Heart J 2015; 36: 1223-30.

8. Mebazaa A, Combes A, van Diepen S, et al. Management of cardiogenic shock complicating myocardial infarction. Intensive Care Med 2018; 44: 760-73.

9. Eltzschig HK, Eckle T. Ischemia and reperfusion-from mechanism to translation. Nat Med 2011; 17: 1391-401.

10. Ibáñez B, Heusch G, Ovize M, Van De Werf F. Evolving therapies for myocardial ischemia/reperfusion injury. J Am Coll Cardiol 2015; 65: 1454-71.

11. Turer AT, Hill JA. Pathogenesis of myocardial ischemia-reperfusion injury and rationale for therapy. Am J Cardiol 2010; 106: 360-8.

12. Xie N, Zhang W, Li J, et al. $\alpha$-Linolenic acid intake attenuates myocardial ischemia/reperfusion injury through anti-inflammatory and anti-oxidative stress effects in diabetic but not normal rats. Arch Med Res 2011; 42 171-81.

13. Yun N, Kang JW, Lee SM. Protective effects of chlorogenic acid against ischemia/reperfusion injury in rat liver: molecular evidence of its antioxidant and anti-inflammatory properties. J Nutr Biochem 2012; 23: 1249-55.

14. Kim YM, Ha YM, Jin YC, et al. Palmatine from Coptidis rhizoma reduces ischemia-reperfusion-mediated acute myocardial injury in the rat. Food Chem Toxicol 2009; 47: 2097-102.

15. Moskalenko AV. Nonlinear nature of the antiarrhythmic effects of lidocain. Biofizika 2009; 54: 62-7.

16. Campbell RWF, Dolder MA, Prescott LF, et al. Comparison of procainamide and mexiletine in prevention of ventricular arrhythmias after acute myocardial infarction. Lancet 1975; 305: 1257-60.

17. Quevedo M, Perez-Olea J, Pino C. Prilocaine effect on aconitine-induced arrhythmias. Acta Physiol Latino-Americana 1979; 28: 201-7.

18. Picard S, Rouet R, Flais F, et al. Proarrhythmic and antiarrhythmic effects of bupivacaine in an in vitro model of myocardial ischemia and reperfusion. Anesthesiology 1998; 88: 1318-29.

19. Marwick PC, Levin Al, Coetzee AR. Recurrence of cardiotoxicity after lipid rescue from bupivacaine-induced cardiac arrest. Anesth Analg 2009; 108: 1344-6.

20. Graf BM, Abraham I, Eberbach N, et al. Differences in cardiotoxicity of bupivacaine and ropivacaine are the result of physicochemical and stereoselective properties. Anesthesiology 2002; 96: 1427-34.

21. Nguyen PT, DeMarco KR, Vorobyov I, et al. Structural basis for antiarrhythmic drug interactions with the human cardiac sodium channel. Proc Natl Acad Sci USA 2019; 116: $2945-54$
22. Borgeat A, Aguirre J. Update on local anesthetics. Curr Opin Anaesthesiol 2010; 23: 466-71.

23. Patel N. Ropivacaine. In: The Essence of Analgesia and Analgesics. Sinatra RS, Jahr JS. University of California, Los Angeles, School of Medicine, J. Michael Watkins-Pitchford, Cambridge University Press 2010; 276-9.

24. Ruetsch Y, Boni T, Borgeat A. From cocaine to ropivacaine: the history of local anesthetic drugs. Curr Top Med Chem 2005; 1: 175-82.

25. Piegeler T, Votta-Velis EG, Bakhshi FR, et al. Endothelial barrier protection by local anesthetics: ropivacaine and lidocaine block tumor necrosis factor-alpha-induced endothelial cell Src activation. Anesthesiology 2014; 120: 1414-28.

26. Blumenthal S, Borgeat A, Pasch T, et al. Ropivacaine decreases inflammation in experimental endotoxin-induced lung injury. Anesthesiology 2006; 104: 961-9.

27. Piegeler T, Dull RO, Hu G, et al. Ropivacaine attenuates endotoxin plus hyperinflation-mediated acute lung injury via inhibition of early-onset Src-dependent signaling. BMC Anesthesiol 2014; 14: 57.

28. Gong X, Dan J, Li F, Wang L. Suppression of mitochondrial respiration with local anesthetic ropivacaine targets breast cancer cells. J Thorac Dis 2018; 10: 2804-12.

29. Baptista-Hon DT, Robertson FM, Robertson GB, et al. Potent inhibition by ropivacaine of metastatic colon cancer SW620 cell invasion and NaV1.5 channel function. $\mathrm{Br} J$ Anaesth 2014; 113: 138-9.

30. Lirk P, Hollmann MW, Fleischer M, et al. Lidocaine and ropivacaine, but not bupivacaine, demethylate deoxyribonucleic acid in breast cancer cells in vitro. $\mathrm{Br} J$ Anaesth 2014; 113: 132-8.

31. Piegeler T, Votta-Velis EG, Liu G, et al. Antimetastatic potential of amide-linked local anesthetics: inhibition of lung adenocarcinoma cell migration and inflammatory Src signaling independent of sodium channel blockade. Anesthesiology 2012; 117: 548-59.

32. Xu Q, Li QG, Fan GR, et al. Protective effects of fentanyl preconditioning on cardiomyocyte apoptosis induced by ischemia-reperfusion in rats. Braz J Med Biol Res 2017; 50: e5286.

33. Moraes ADT de O, Miranda MDS de, Jacob ÍTT, et al. Synthesis, in vitro and in vivo biological evaluation, COX-1/2 inhibition and molecular docking study of indole-N-acylhydrazone derivatives. Bioorganic Med Chem 2018; 26: 5388-96.

34. Booth EA, Obeid NR, Lucchesi BR. Activation of estrogen receptor-alpha protects the in vivo rabbit heart from ischemia-reperfusion injury. Am J Physiol Hear Circ Physiol 2005; 289: H2039-47.

35. Lagranha CJ, Deschamps A, Aponte A, et al. Sex differences in the phosphorylation of mitochondrial proteins result in reduced production of reactive oxygen species and cardioprotection in females. Circ Res 2010; 106: 1681-91.

36. Talukder MAH, Yang F, Shimokawa H, Zweier JL. eNOS is required for acute in vivo ischemic preconditioning of the heart: effects of ischemic duration and sex. Am J Physiol Hear Circ Physiol 2010; 299: H437-45.

37. Timmers L, Lim SK, Arslan F, et al. Reduction of myocardial infarct size by human mesenchymal stem cell conditioned medium. Stem Cell Res 2008; 1: 129-37.

38. García-Prieto J, Villena-Gutiérrez R, Gómez M, et al. Neutrophil stunning by metoprolol reduces infarct size. Nat Commun 2017; 8: 14780.

39. Albers GW, Goyal M, Jahan R, et al. Ischemic core and hypoperfusion volumes predict infarct size in SWIFT PRIME. Ann Neurol 2016; 79: 76-89. 
40. Stub D, Smith K, Bernard S, et al. Air versus oxygen in ST-segment-elevation myocardial infarction. Circulation 2015; 131: 2143-50.

41. Vogel B, Claessen BE, Arnold SV, et al. ST-segment elevation myocardial infarction. Nat Rev Dis Prim 2019; 5: 39.

42. Abushouk Al, Ismail A, Salem AMA, et al. Cardioprotective mechanisms of phytochemicals against doxorubicin-induced cardiotoxicity. Biomed Pharmacother 2017; 90: 935-46.

43. Kulczyński B, Gramza-Michałowska A, Kobus-Cisowska J, Kmiecik D. The role of carotenoids in the prevention and treatment of cardiovascular disease - current state of knowledge. J Funct Foods 2017; 38: 45-65.

44. Nabati M, Janbabai G, Baghyari S, et al Cardioprotective effects of carvedilol in inhibiting doxorubicin-induced cardiotoxicity. J Cardiovasc Pharmacol 2017; 69: 279-85.

45. Zeng C, Zhong P, Zhao Y, et al. Curcumin protects hearts from FFA-induced injury by activating Nrf2 and inactivating NF- B both in vitro and in vivo. J Mol Cell Cardiol 2015; 79: 1-12.

46. Qin C, Yang YH, May L, et al. Cardioprotective potential of annexin-A1 mimetics in myocardial infarction. Pharmacol Ther 2015; 148: 47-65.

47. Jokerst JV, Cauwenberghs N, Kuznetsova T, et al. Circulating biomarkers to identify responders in cardiac cell therapy. Sci Rep 2017; 7: 4419.

48. Reinstadler SJ, Feistritzer HJ, Reindl $M$, et al. Combined biomarker testing for the prediction of left ventricular remodelling in ST-elevation myocardial infarction. Open Hear 2016; 3: e000485.

49. Bernat-Adell MD, Collado-Boira EJ, Moles-Julio P, et al Recovery of inflammation, cardiac, and muscle damage biomarkers after running a marathon. J Strength Cond Res 2021; 35: 626-32.

50. Dai DF, Chiao Y, Marcinek DJ, et al. Mitochondrial oxidative stress in aging and healthspan. Longev Heal 2014; 3: 6 .

51. Chen QM, Morrissy S, Alpert JS. Oxidative Stress and Heart Failure. In: Comprehensive Toxicology. Third Edition. The University of Arizona 2018; 230-51.

52. Mei Y, Thompson MD, Cohen RA, Tong XY. Autophagy and oxidative stress in cardiovascular diseases. Biochim Biophys Acta Mol Basis Dis 2015; 1852: 243-51.

53. Hussain T, Tan B, Yin Y, et al. Oxidative stress and inflammation: what polyphenols can do for us? Oxid Med Cell Longev 2016; 2016: 7432797.

54. Mittal M, Siddiqui MR, Tran K, et al. Reactive oxygen species in inflammation and tissue injury. Antioxidants Redox Signal 2014; 20: 1126-67.

55. Chiong M, Wang ZV, Pedrozo Z, et al. Cardiomyocyte death: mechanisms and translational implications. Cell Death Dis 2011; 2: e244.

56. Barile L, Lionetti V, Cervio E, et al. Extracellular vesicles from human cardiac progenitor cells inhibit cardiomyocyte apoptosis and improve cardiac function aftermyocardial infarction. Cardiovasc Res 2014; 103: 530-41.

57. Shinmura K, Xuan YT, Tang XL, et al. Inducible nitric oxide synthase modulates cyclooxygenase- 2 activity in the heart of conscious rabbits during the late phase of ischemic preconditioning. Circ Res 2002; 90: 602-8.

58. Nan J, Hu H, Sun Y, et al. TNFR2 stimulation promotes mitochondrial fusion via Stat3- and NF-kB-dependent activation of OPA1 expression. Circ Res 2017; 121: 392-410.

59. Singh UP, Bhat HR, Gahtori P, Singh RK. Hybrid phenylthiazole and 1,3,5-triazine target cytosolic leucyl-tRNA synthetase for antifungal action as revealed by molecular docking studies. Silico Pharmacol 2013; 1: 3.
60. Srivastava JK, Pillai GG, Bhat HR, et al. Design and dis covery of novel monastrol-1,3,5-triazines as potent anti-breast cancer agent via attenuating epidermal growth factor receptor tyrosine kinase. Sci Rep 2017; 7: 5851.

61. Masih A, Agnihotri AK, Srivastava JK, et al. Discovery of novel pyrazole derivatives as a potent anti-inflammatory agent in RAW264.7 cells via inhibition of NF-KB for possible benefit against SARS-CoV-2. J Biochem Mol Toxicol 2020; doi: 10.1002/jbt.22656.

62. Singh UP, Bhat HR, Verma A, et al. Phenyl hydrazone bearing pyrazole and pyrimidine scaffolds: design and discovery of a novel class of non-nucleoside reverse transcriptase inhibitors (NNRTIs) against HIV-1 and their antibacterial properties. RSC Adv 2013; 3: 17335-48.

63. Singh UP, Singh RK. Molecular docking analysis of novel non-nucleoside reverse transcriptase inhibitors in development: implication for rational drug design. Retrovirology 2011; 8: P82. 